\title{
AUTOMATIC CALIBRATION OF A WAVE MODEL WITH AN EVOLUTIONARY BAYESIAN METHOD
}

\author{
Rodrigo Alonso ${ }^{1}$ and Sebastián Solari ${ }^{1}$
}

\begin{abstract}
Bayesian Inference has been widely applied with success in science and engineering. One of its main uses is the inference of model parameters in order to reconcile model outputs with evidence provided by measures. In this article we propose this application for coastal engineering problems. Specifically, it is proposed to infer the parameters of a numerical wave model used to downscale wave reanalysis data to a coastal site. The proposed method is applied to a case study on the Uruguayan Atlantic coast, where a few month wave measure data series is available and needs to be extended in order to be used on an engineering project. The wave model used is SWAN and the data in deep waters and the wind data were obtained from the ERA-Interim reanalysis. At first, the method was tested with one and two parameters, since in these cases it is possible to compare the obtained results with a plot of the target function. Finally it was used to calibrate four parameters of the wave model and assess the uncertainty introduced by the selection of a set of parameters.
\end{abstract}

Keywords: Bayesian inference; wave modelling; wave reanalysis downscaling; Markov chain Monte Carlo

\section{INTRODUCTION}

Long-term and good quality wave data series are required for any coastal engineering project. But reliable coastal wave data is only available in a few places, where long-term wave measurements are available and have been used for calibration and validation of high-quality and high-resolution wave hindcasts. For most regions of the world, typically in developing countries, only global reanalysis wave data is available (e.g. ERA-Interim), usually in combination with a short-term (i.e. several month) insitu measured series obtained for a particular project.

The spatial resolution of global wave reanalysis is not enough for coastal applications; moreover, nearshore wave transformation processes are not properly accounted for. Therefore, wave data from global reanalysis must be transferred to the coast in a proper way before being used in coastal projects (i.e. data must be downscaled). Some methodologies have been developed for efficiently downscale wave data (e.g. Camus et al. 2011) that are based on the use of third generation coastal wave models (e.g. SWAN; Booij et al. 1999). These methods are known as physical downscaling, as opposed to statistical ones, and they have two basic steps: 1) the model calibration problem and 2) perform the long-term simulation with the calibrated model.

While the use of third generation wave models with off-shore global wave reanalysis data as boundary condition, is widespread in coastal applications, there is no consensus about how these models should be calibrated. To the best of our knowledge, these issues are addressed with ad hoc approaches developed for each project. As a model like SWAN has numerous parameters, almost one for each source term of the wave action balance equation, the manual adjustment of them becomes labor-intensive and strongly dependent on the modeler. For that reasons, an automatic calibration method seems to be very helpful.

In addition to automatic calibration, methods to assess the uncertainty introduced by the assignation of values to model parameters are also necessary. This is one of the main sources of uncertainty of model outputs, so it should be assessed in order to be included on the probabilistic design methods that coastal engineering projects requires. For that reason, it is considered important to search for new methodologies that could provide more accurate and complete estimations of uncertainties.

In this paper, we propose the use of Bayesian methodologies to deal with the calibration of a third generation wave model. This kind of methods allow to efficiently handle a large number of parameters in the calibration, also providing an estimation of the joint probability distribution of these parameters, allowing to quantify the uncertainty of the model that is related with these parameters. At the same time, they have many positive precedents addressing similar problems as the described before but in other areas (e.g. Vrugt et al. 2003, 2008 and 2016)

The method is applied to a case study in the Uruguayan Atlantic coast where a few month wave measure data series is available and needs to be extended in order to be used on an engineering project. The wave model used is SWAN (Booij et al. 1999) and waves in deep waters and the wind data were

\footnotetext{
${ }^{1}$ Instituto de Mecánica de los Fluidos e Ingeniería Ambiental, Facultad de Ingeniería, Universidad de la República, Julio Herrera y Reissig 565, Montevideo, 11300, Uruguay.
} 
obtained from the ERA-Interim reanalysis (Dee et al. 2011). At first, the method was tested with one and two parameters, since in these cases it is possible to compare the obtained results with a plot of likelihood estimation vs. parameters values (i.e. target function used to calibrate). Finally the proposed method was used to calibrate four parameters of the wave model and assess the uncertainty introduced by the selection of the obtained set of parameters.

The paper is organized as follows. The first section after introduction, briefly describe the theoretical framework necessary to understand the method. Basic ideas of Bayesian inference are followed by a general description of the Markov chain Monte Carlo algorithms (MCMC) and particularly the one that was used which name is Differential Evolution Adaptative Metropolis (DREAM) and was developed by Vrugt et al. $(2008,2009)$. Then the application to the calibration problem involved in the wave reanalysis downscaling to a coastal site of Uruguay is presented, to end summarizing the main conclusions and exposing future steps planned to go ahead exploring the potential of Bayesian inference on coastal engineering applications.

\section{THEORETICAL FRAMEWORK}

\section{Bayesian Inference}

Bayesian inference is a method of statistical inference in which Bayes theorem is used to update the probability for a hypothesis as more evidence becomes available. In the case of a model calibration problem, the hypothesis is a set of values of model parameters and the evidence are measures that could be compared with model outputs.

In the Bayesian approach parameters are represented with a probability density function (pdf). It does not mean that we believe that values of unknown parameters are random; it only means that our knowledge of a parameter's value is uncertain, and that our uncertainty about this value can be represented using an appropriate pdf (Hamada et al. 2008).

If $\theta$ and $y$ are the parameter and observation vectors respectively, Bayes theorem is applied as follows, involving four pdf's,

$$
p(\theta / y)=\frac{p(y / \theta) \cdot p(\theta)}{p(y)}
$$

where $\mathrm{p}(\theta / y)$ is called the posterior distribution and it is what we are looking for, while $\mathrm{p}(\theta)$ is the prior distribution and reflects our belief before observed data are analyzed. On the other hand, $\mathrm{p}(y / \theta)$ is the likelihood function that could be estimated assuming certain behavior of the model errors (e.g. Gaussian distributed) and $\mathrm{p}(y)$ is the marginal distribution of the observations. In practice, $\mathrm{p}(y)$ is not required for posterior estimation as all statistical inferences about $\mathrm{p}(\theta / y)$ can be made from the unnormalized density,

$$
\text { posterior } \alpha \text { likelihood x prior }
$$

The reality is that for most of the real-world problems the posterior distribution cannot be obtained by analytical approximation. For that reason, Bayesian approach was not widely used before the popularization of computational methods. But while computer became more powerful algorithms like Markov chain Monte Carlo (MCMC) allowed to extend its applications to diverse, complex and multiparametric problems, by estimating the posterior pdf from a sample generated by the algorithm.

\section{MCMC algorithms}

MCMC algorithms are a general class of computational methods used to generate samples from posterior distributions. The desired summary of the posterior distribution is then obtained from the sample. The posterior distribution, also referred to as the target distribution is often high-dimensional. Since their introduction in the 1990's, they have been successfully applied to thousands of applications (Hamada et al. 2008).

MCMC algorithms produce random walks over a probability distribution. By taking a sufficient number of steps in this random walk, the MCMC simulation algorithm visits various regions of the parameter space in proportion to their posterior probabilities. In other words, it is a stochastic simulation that successively visits solutions in the parameter space with stable frequencies stemming from a fixed probability distribution.

MCMC algorithms are classified in two categories: Metropolis-Hastings algorithms and Gibbs samplers. The algorithm used in the present work belongs to the first group. These kinds of algorithms 
have three basic steps. The first is the generation of a candidate point $\theta^{*}$. This requires a sampler or proposal distribution. The second step is the computation of the acceptance probability $r$,

$$
r=\min \left\{1, \frac{p\left(\theta^{*}\right)}{p\left(\theta^{t-1}\right)}\right\}
$$

Where $\theta^{t-1}$ is the actual state of the chain and $\mathrm{p}\left(\theta^{t-1}\right)$ denotes the probability to find the system there. In the same way, $\mathrm{p}\left(\theta^{*}\right)$ is the probability to find the system at the candidate point. Finally, $\mathrm{r}$ is compared with a random variable $u$, uniformly distributed between 0 and 1 ( $\left.u \sim \mathscr{U}\left[\begin{array}{ll}0 & 1\end{array}\right]\right)$. If $\mathrm{r}$ is equal or greater than $u$, the sequence moves from $\theta^{t-1}$ to $\theta^{*}$. Otherwise the sequence is kept in $\theta^{t-1}$

MCMC requires the choice of a proposal distribution to generate transitions in the Markov chain. This choice is therefore crucial and determines the practical applicability of MCMC in many fields of study (Owen and Tribble, 2005). To improve the search efficiency of MCMC samplers it seems natural to tune the proposal distribution during the evolution to the posterior target distribution, using information inferred from the sampling history induced by the transitions of the Markov chain (Vrugt et al 2003). In the past decade many adaptive samplers were proposed in order to improve MCMC simulations, one of those that has given satisfactory results in various applications, and has the support of a mathematical proof of convergence, is the DREAM algorithm. It is the one used in this work and it is briefly described in the next sub-section.

\section{DREAM algorithm}

Its name comes from DiffeRential Evolution Adaptative Metropolis (DREAM). It was developed by Vrugt et al. (2008 and 2009). It is a multiple chain method that uses differential evolution as genetic algorithm for population evolution with a Metropolis selection rule to decide whether candidate points should replace their parents or not.

This multi-chain MCMC algorithm automatically tunes the scale and orientation of the proposal distribution en route to the target distribution. Maintaining detailed balance and ergodicity. It has showed excellent performance on complex, multimodal search problems. Its proof of convergence to the posterior distribution in ter Braak and Vrugt (2008).

Since its appearance, different variants have been proposed, forming the DREAM family of algorithms. Vrugt (2016) presents the distinctive features of each one. The one used in this work is $\operatorname{DREAM}_{(\mathrm{ZS})}$.

\section{APPLICATION}

\section{Study zone}

The DREAM $(\mathrm{ZS})$ algorithm was applied to a case study in the Uruguayan Atlantic coast where a few month wave measure data series is available and needs to be extended in order to be used on an engineering project. To obtain the long-term wave data series, a physical downscaling of the reanalysis ERA-Interim to the coastal site was planned. The algorithm was applied to calibrate, using measured data, the parameters of the wave model implemented to downscale reanalysis data and to assess the uncertainty introduced by model parameters

Observations were collected with an acoustic Doppler current profiler (ADCP) installed on a water depth of $18 \mathrm{~m}$ in the site indicated in Figure 1. In that figure is also appreciated the closest ERA Interim node $(-53 \mathrm{~W}-35 \mathrm{~S})$, located $100 \mathrm{~km}$ offshore, on a water depth of $60 \mathrm{~m}$. The observation series has data every 3 hs, covering the period October 2013 - April 2014. While Reanalysis data has a time step of 6 hs $(00,06,12,18$ UTC) starting on 1979 and continuing until present. Wind data at $10 \mathrm{~m}$ height used as model input was also obtained from ERA-Interim. 


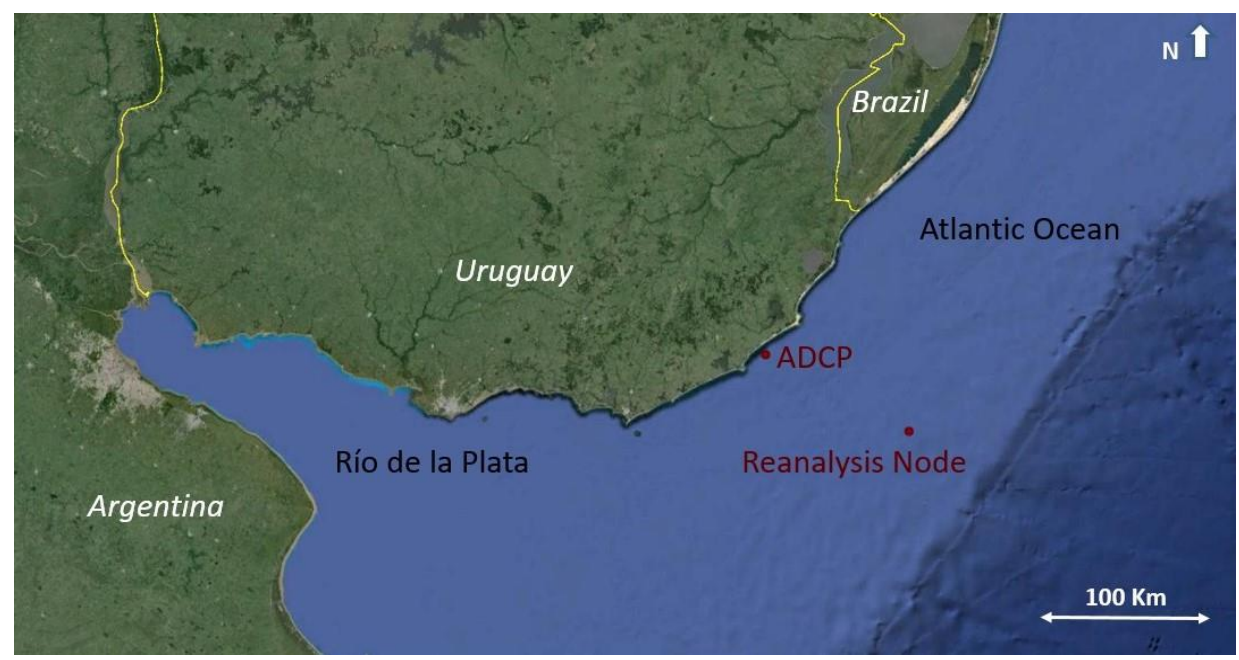

Figure 1. Study zone. Locations of ADCP and the closest ERA-Interim node.

\section{Wave model}

Wave simulations were performed with the third generation wave numerical model SWAN (Booij et al. 1999). It is a numerical model widely used by the coastal engineering community. It solves the wave action balance equations allowing wave spectra to evolve without restrictions.

The domain and computational grid are shown in Figure 2. The rectangular and regular computational grid is $279.2 \mathrm{~km}$ long in the alongshore direction with a mesh size $(\Delta \mathrm{x})$ of $10 \mathrm{~km}$, while in the cross-shore direction it is $127.1 \mathrm{~km}$ long with a with a mesh size $(\Delta y)$ of $5 \mathrm{~km}$. The grid width was defined wide enough so that boundary conditions at lateral borders did not affect model outputs at the point of interest. Regarding to mesh sizes and taking into account that Monte Carlo algorithms requires many simulations, they were defined in such a way that the simulations became as fast as possible without affecting the results at the point of interest. In the same way, quasi-steady runs were performed so for the scale of the problem, model results do not differ with those of unsteady simulations and quasi-steady runs are much faster.
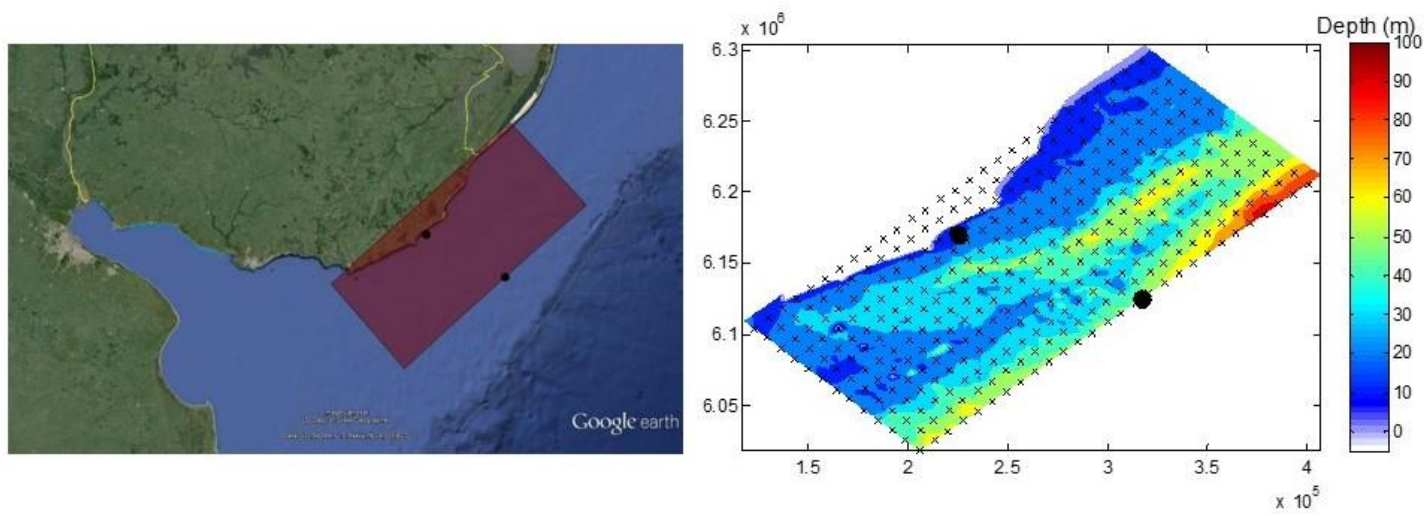

Figure 2. Domain (left) and computational grid (right) of the wave model. The black dots are the ADCP location and the ERA-Interim node.

\section{Calibration parameters}

Initially sensitivity analysis of significant wave height (Hs) model results at the ADCP point to each source were performed. It was observed that the source terms that have significant impact on Hs results at ADCP point are bottom friction $\left(S_{b o t}\right)$ and whitecapping dissipation $\left(S_{d s}\right)$. This is shown in the graphs of Figure 3. 

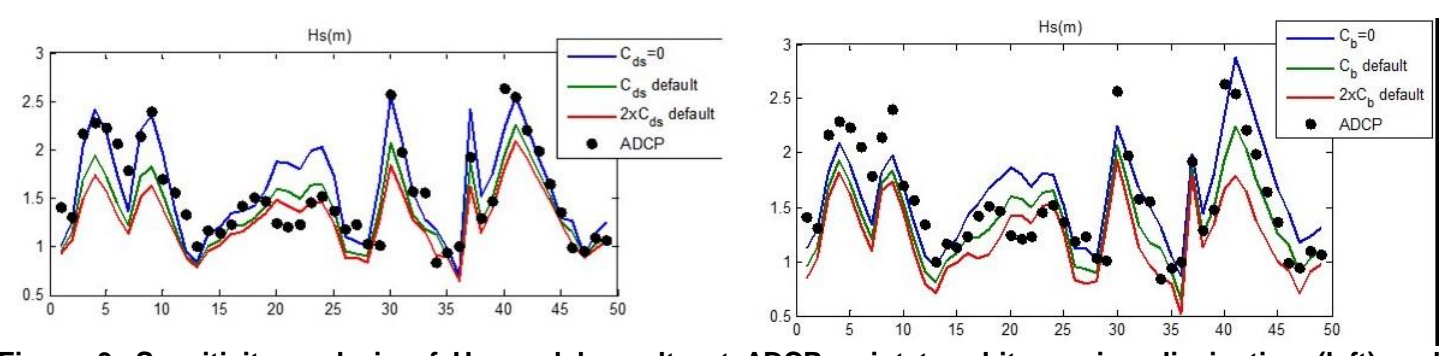

Figure 3. Sensitivity analysis of Hs model results at ADCP point to whitecapping dissipation (left) and bottom friction (right). Hs in meters at ADCP are in the $y$ axis, while the number of the event is in the $x$ axis. (50 consecutives events were simulated with a quasi-steady run).

Based on this results, we opted to focus the calibration on the parameters involved in the parametrization of these terms. The bottom friction parametrization correspond to the empirical model of JONSWAP (Hasselmann et al. 1973), and it is expressed in the following form:

$$
S_{b o t}(\sigma, \theta)=-C_{b} \frac{\sigma^{2}}{g^{2}(\sinh (k d))^{2}} E(\sigma, \theta)
$$

Where $\sigma, k$ and $\theta$ are the frequency, wave number and direction of the wave spectrum bin whose energy is $E(\sigma, \theta), g$ is the gravitational acceleration, $S_{b o t}(\sigma, \theta)$ is energy dissipated (negative value) by bottom friction on the spectrum bin defined by $\sigma$ and $\theta$, while $C_{b}$ is a dimensionless parameter that need to be calibrated.

The whitecapping dissipation parametrization correspond to the pulse-based model of Hasselmann (1974), reformulated in terms of wave number (the WAMDI group, 1988). This expression is:

$$
S_{d s}(\sigma, \theta)=-\Gamma \tilde{\sigma} \frac{k}{\tilde{k}} E(\sigma, \theta)
$$

and the expression of $\Gamma$ is,

$$
\Gamma=-C_{d s}\left((1-\delta)+\delta \frac{k}{\bar{k}}\right)\left(\frac{\tilde{s}}{\tilde{s}_{P M}}\right)^{p}
$$

$S_{d s}(\sigma, \theta)$ is energy dissipated by whitecapping on the spectral bin $(\sigma, \theta), \tilde{\sigma}, \tilde{k}$ and $\tilde{s}$ are the mean frequency, the mean wave number and the overall wave steepness respectively. $\tilde{s}_{P M}$ is the value of $\tilde{s}$ for the Pierson Moskowitz spectrum (1964). While $C_{d s}, \delta$ and $p$ are tuneable dimensionless parameters.

At first, DREAM (zs) algorithm was tested with one $\left(C_{b}\right)$ and two parameters: $C_{b}$ and $C_{d s}$. Since in these cases it is possible to compare the obtained results with a plot of likelihood estimation vs. parameters values. Then the proposed method was used to calibrate the four parameters: $C_{b}, C_{d s}, \delta$ and $p$.

Results.

The target function used in all cases is an error measurement of the significant wave height. It was estimated assuming that errors are independent and identically distributed with a zero mean normal distribution. With these assumptions the expressions is as follows:

$$
\log \text { likelihood }=-\frac{1}{2} \times N \times \log \left(\sum_{i=1}^{N}\left(H_{s, i}^{\bmod e l}-H_{s, i}^{o b s}\right)^{2}\right)
$$

Where $\mathrm{N}$ is the amount of data compared, $H s_{i}^{\text {model }}$ and $H s_{i}^{o b s}$ correspond to the significant wave heights estimated and measured respectively.

For application of DREAM (Zs) in all presented cases 3 chains were considered, while 4000 evolutions were allowed for each chain, monitoring convergence with the R-statistics of Gelman and Rubin (1992).

1 Parameter. DREAM (zS) was initially applied to calibrate only the parameter of the bottom friction parametrization $\left(C_{b}\right)$. Figure 4 shows the sample obtained with the Markov chain, the posterior 
distribution of $C_{b}$ summarized as a histogram, and the evolution of the R statistcs used to monitor convergence. In the later is observed that the simulation converge rapidly. In addition Figure 5 shows a comparison between the results obtained with the method, summarized on the mean value, percentile $5 \%$ and $95 \%$, with a plot of likelihood estimation (See eq. 7) vs. $C_{b}$. In this figure it is appreciated the effectiveness of the method to find the optimum of the target function.
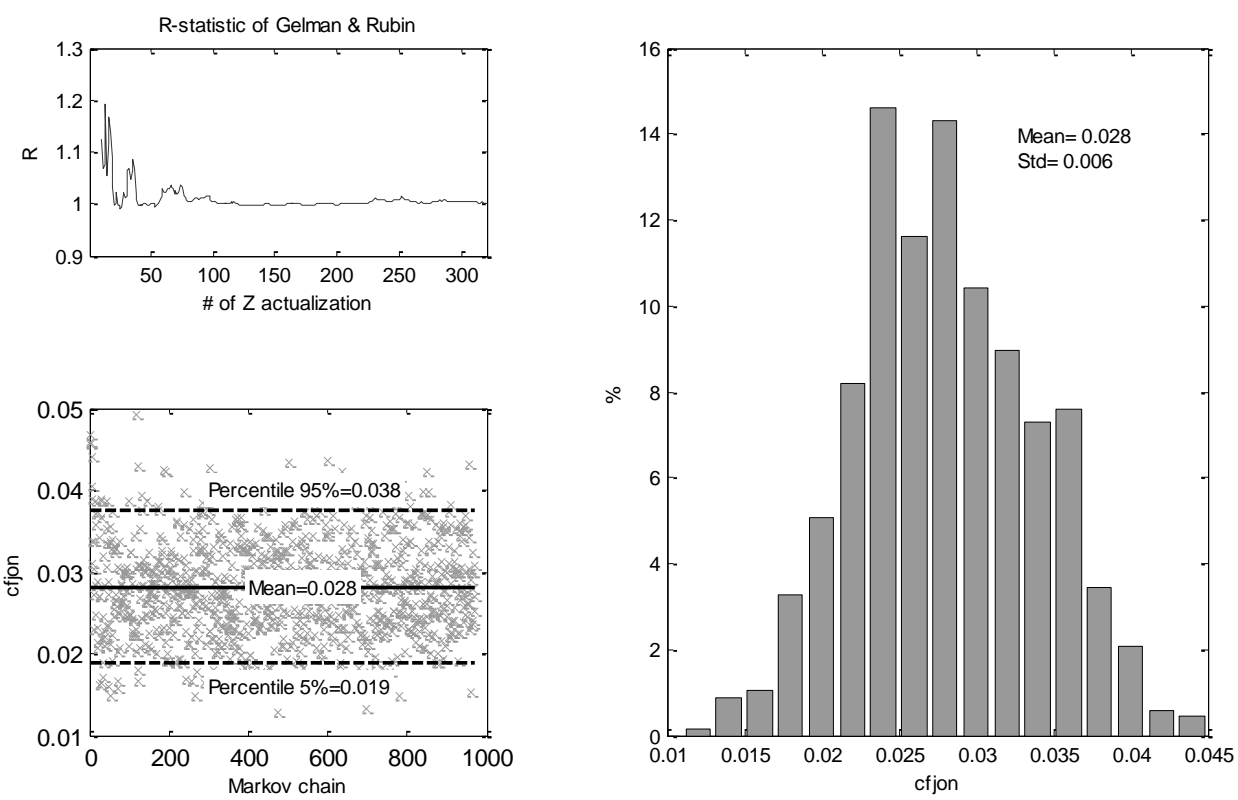

Figure 4. Test with one parameter. Markov chain evolution (lower and left), R-statistic evolution (upper and left) and histogram of $C_{b}$ (right). Observation: cfjon is the same as $C_{b}$ defined in the text.

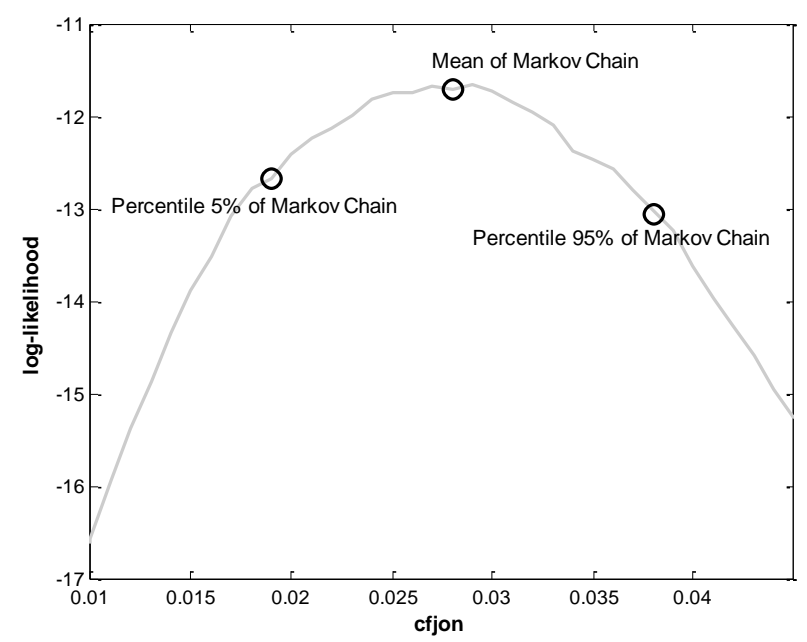

Figure 5. Comparison between the posterior distribution of $C_{b}$ obtained with the method and the plot likelihood vs. $C_{b}$ (or cfjon).

It is also remarkable the proximity with the optimum that presents the SWAN default value of $C_{b}$ $\left(C_{b}=0.038\right)$.

2 Parameters. DREAM (zs) was applied to calibrate two parameters together: $C_{b}$ and $\mathrm{C}_{\mathrm{ds} .}$. Figure 6 shows the comparison between the obtained sample of parameters values and the surface plot of likelihood vs parameters values. It is observed again the ability of the algorithm to find the optimum of 
the target function. And SWAN default values of parameters $\left(C_{b}=0.038, C_{d s}=2.36 \times 10^{-5}\right)$ are again very close to the optimum.

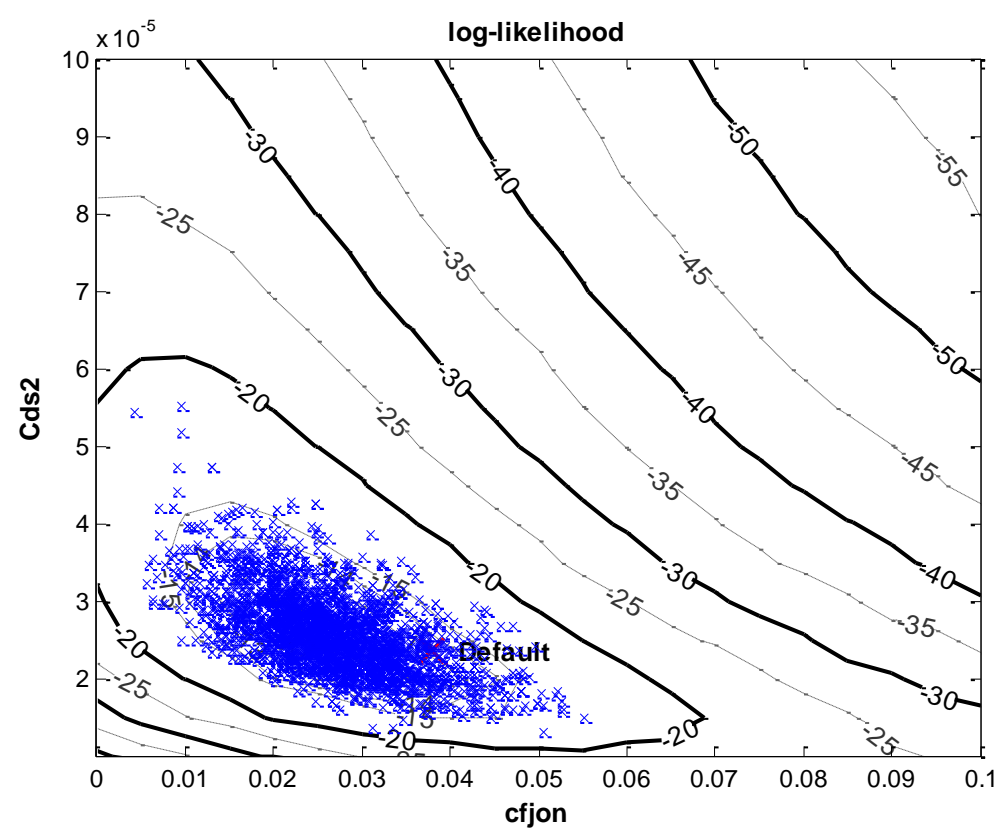

Figure 6. Comparison between the Markov chain and the surface plot: likelihood vs parameters values.

4 Parameters. Finally the method was applied to calibrate the four parameters: $C_{b}, C_{d s}, \delta$ and $p$. Figures 7 to 10 shows the Markov chain and posterior distribution summarized by a histogram for each of the parameters. While tables 1 to 3 shows the model performance with the calibrated parameters values (i.e the mean of the obtained sample) and with the default SWAN parameters values. The error metric considered are: root mean square error (RMSE), BIAS, correlation coefficient ( $r$ ) and Scatter Index (SI). While the wave parameters shown are Hs (Table 1), period Tm01 (Table 2) and mean direction Dm (Table 3).
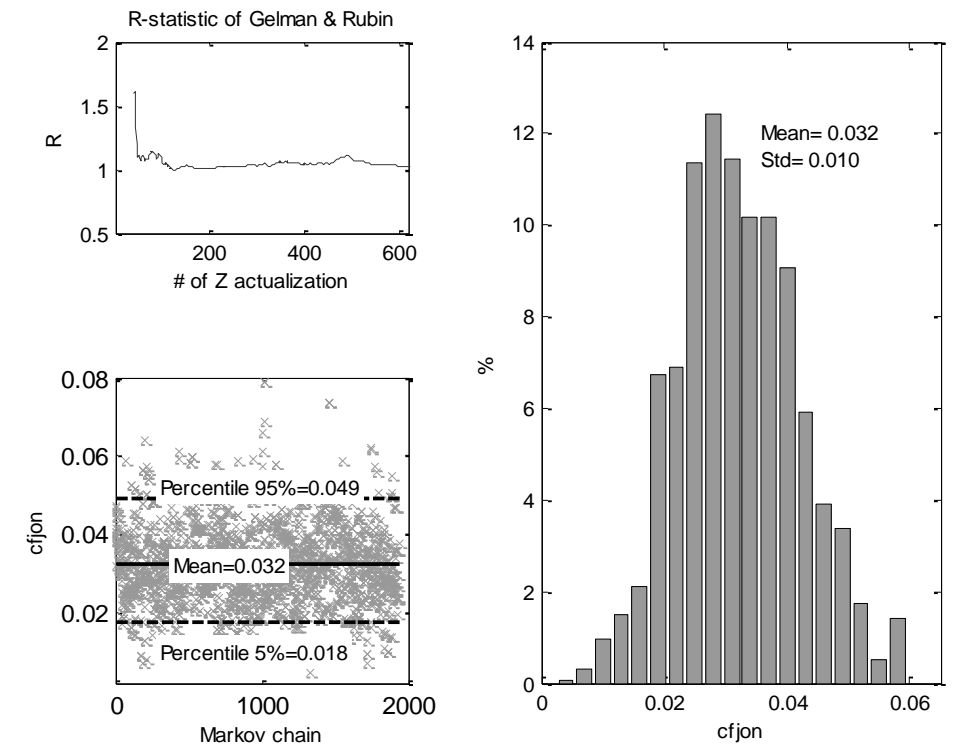

Figure 7. Markov chain evolution (lower and left), R-statistic evolution (upper and left) and histogram of $C_{b}$ (right). 

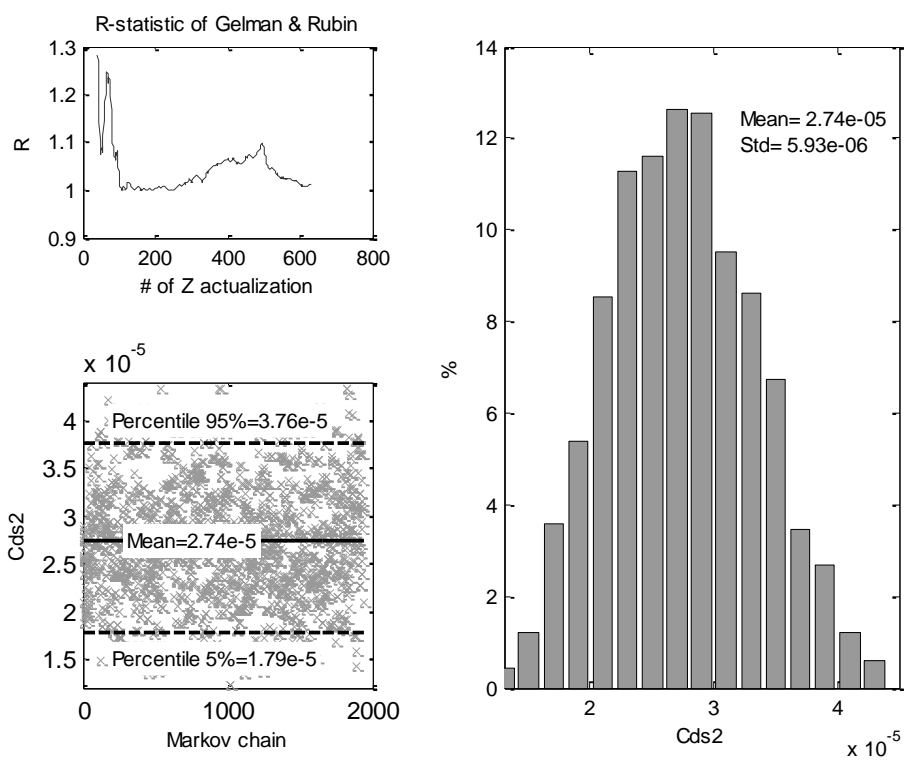

Figure 8. Markov chain evolution (lower and left), R-statistic evolution (upper and left) and histogram of $C_{d s}$ (right).
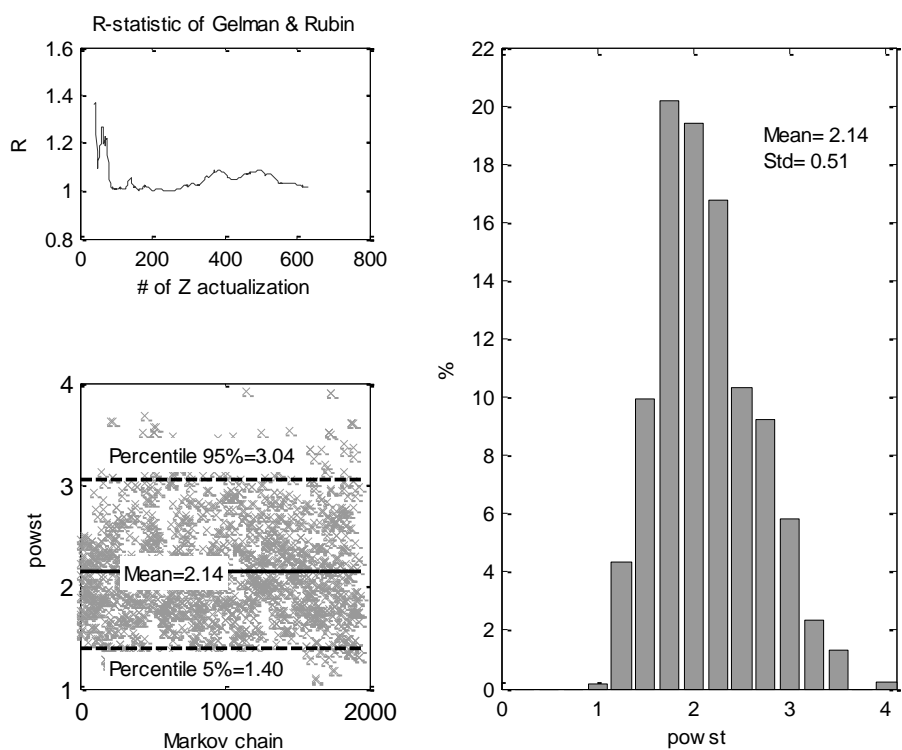

Figure 9. Markov chain evolution (lower and left), R-statistic evolution (upper and left) and histogram of $p$ (right). 

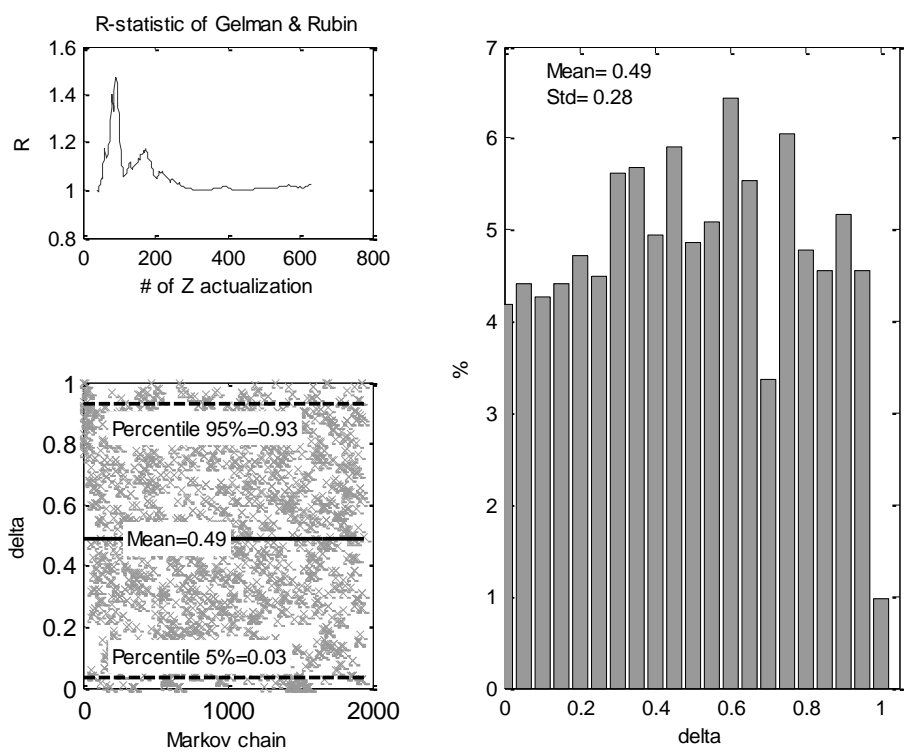

Figure 10. Markov chain evolution (lower and left), R-statistic evolution (upper and left) and histogram of $\delta$ (right).

All the graphics of the evolutions of $\mathrm{R}$ statistics denote a rapidly convergence of the simulation. The obtained histograms have a reasonable form, with small variance for the case of $C_{b}, C_{d s}$ and $p$, while $\delta$ seems to adjust to a uniform pdf, which would imply that its value has minor incidence in the analyzed model results, so it could not have been considered in the calibration.

\begin{tabular}{|c|c|c|c|c|}
\hline & BIAS (m) & RMSE (m) & $r$ & $\mathrm{SI}$ \\
\hline $\begin{array}{l}\text { Default } \\
\text { Calibrated }_{5}\end{array}$ & $\begin{array}{l}-0.1 \\
-0.03\end{array}$ & $\begin{array}{l}0.25 \\
0.23\end{array}$ & $\begin{array}{l}0.86 \\
0.87 \\
\end{array}$ & $\begin{array}{l}18.9 \\
18.6 \\
\end{array}$ \\
\hline
\end{tabular}

\begin{tabular}{|c|c|c|c|c|}
\hline & BIAS (s) & RMSE (s) & $r$ & $\mathrm{SI}$ \\
\hline $\begin{array}{l}\text { Default } \\
\text { Calibrated }_{5}\end{array}$ & $\begin{array}{l}-1.3 \\
-1.8\end{array}$ & $\begin{array}{l}1.6 \\
2.0\end{array}$ & $\begin{array}{l}0.62 \\
0.61\end{array}$ & $\begin{array}{l}15.8 \\
15.0\end{array}$ \\
\hline
\end{tabular}

\begin{tabular}{|c|c|c|c|c|}
\hline & BIAS (ํ) & RMSE (ㅇ) & $r$ & $\mathrm{SI}$ \\
\hline $\begin{array}{l}\text { Default } \\
\text { Calibrated }_{5}\end{array}$ & $\begin{array}{l}7.4 \\
6.8\end{array}$ & $\begin{array}{l}34.5 \\
34.8\end{array}$ & $\begin{array}{l}0.65 \\
0.64\end{array}$ & $\begin{array}{l}28.3 \\
28.7\end{array}$ \\
\hline
\end{tabular}

It is observed that the calibrated parameter values improves the Hs model results but that not happens with the other wave parameter that were not considered in the target function.

\section{CONCLUSIONS AND FUTURE WORK}

A first experience of Bayesian inference applied to coastal engineering problems was realized and exposed in this paper. In this first experiment we worked with the model calibration problem involved in the physical downscaling of wave reanalysis data towards a coastal site.

The tests performed for one and two parameters showed the effectiveness of the method to find the optimum of the target function. It is worth mentioning the proximity with this optimum that presents the SWAN default values for these parameters. 
The method applied to four parameters also shows a good performance, improving the model results of $\mathrm{Hs}$ at the point of interest. However it did not allow to improve the models results of others wave parameter that were not considered by the target function (e.g. mean period and mean direction). Since measures of the whole spectra are available, it is proposed to apply the method including the whole spectrum in the target function. We expect that this change will allow an integral improvement of the model results and not only for the estimation of Hs.

Although model parameters is one of the main uncertainty source in the outputs of a model of a nature process, it is not the unique. There are also others such as those related with measure errors of the system response, those related to structural problems of the model and those related with input errors. Precisely the latter seems to be relevant to the problem discussed in this paper. Because large errors in reanalysis data are not possible to correct tuning model parameters. But we think that the proposed method provides the possibility of addressing this problem. The idea in which we are working seeks to introduce corrections parameters of the boundary conditions (reanalysis data) that will be calibrated together with model parameters using the proposed method-

This first auspicious experience encourage the exploration of new applications of Bayesian inference on coastal engineering problems (e.g. coastal morphodynamics, surge tides modeling, etc.). At the same time, set the challenge of develop new algorithms or variations in order to better adapt to the peculiarities of this class of problems.

\section{ACKNOWLEDGMENTS}

The European Center for Medium-Range Weather Forecasts (ECMWF) is acknowledge for providing ERA-Interim surface wind and wave data. Uruguayan Ministry of Transport and Public Works (DNH-MTOP) is acknowledge for partial founding this research. Rodrigo Alonso acknowledges the financial support provided by ANII through the postgraduate scholarships program (POS-NAC2012-1-8936)

\section{REFERENCES}

Booij, N., R. C. Ris, \& L-H. Holthuijsen. 1999. A third-generation wave model for coastal regions: 1. Model description and validation. Journal of Geophysical Research: Oceans, 104(C4), 76497666.

Dee, D. P., Uppala, S. M., Simmons, A. J., Berrisford, P., Poli, P., Kobayashi, S., Andrae, U., Balmaseda, M. A., Balsamo, G., Bauer, P., Bechtold, P., Beljaars, A. C. M., van de Berg, L., Bidlot, J., Bormann, N., Delsol, C., Dragani, R., Fuentes, M., Geer, A. J., Haimberger, L., Healy, S. B., Hersbach, H., Hólm, E. V., Isaksen, L., Kållberg, P., Köhler, M., Matricardi, M., McNally, A. P., Monge-Sanz, B. M., Morcrette, J.-J., Park, B.-K., Peubey, C., de Rosnay, P., Tavolato, C., Thépaut, J.-N. and Vitart, F. 2011, The ERA-Interim reanalysis: configuration and performance of the data assimilation system. Q.J.R. Meteorol. Soc., 137: 553-597.

Gelman, A. G., and D. B. Rubin 1992. Inference from iterative simulations using multiple sequence. Stat. Sci 7, $457-472$

Hamada, M.S, Wilson A. G., Reese C. S. and Martz H. F. 2008. Bayesian Reliability, Springer Series in Statistics, New York, 436 pp.

Hasselmann, K., 1974: On the spectral dissipation of ocean waves due to whitecapping, Bound.-layer Meteor., 6, 1-2, 107-127

Hasselmann, K., T.P. Barnett, E. Bouws, H. Carlson, D.E. Cartwright, K. Enke, J.A. Ewing, H. Gienapp, D.E. Hasselmann, P. Kruseman, A. Meerburg, P. Müller, D.J. Olbers, K. Richter, W. Sell and H. Walden, 1973: Measurements of wind-wave growth and swell decay during the Joint North Sea Wave Project (JONSWAP), Dtsch. Hydrogr. Z. Suppl., 12, A8

Pierson, W.J. and L. Moskowitz, 1964: A proposed spectral form for fully developed wind seas based on the similarity theory of S.A. Kitaigorodskii, J. Geophys. Res., 69, 24, 5181-5190

ter Braak, C.J.F. and J.A. Vrugt 2008. Differential Evolution Markov chain Monte Carlo with snooker updater. Stat Comput, 18, 435-446.

Vrugt , J.A., H. V. Gupta, W. Bouten and S. Sorooshian. 2003. A Shuffled Complex Evolution Metropolis algorithm for optimization and uncertainty assessment of hydrologic model parameters. Water Resource Research, 39, NO. 8, 1201.

Vrugt, J.A.,C.J.F. ter Braak, M.P. Clark, J.M. Hyman and B.A. Robinson. 2008. Treatment of input uncertainty in hydrologic modeling: Doing hydrology backward with Markov chain Monte Carlo simulation. Water Resource Research, 44, W00B09. 
Vrugt , J.A.,C.J.F. ter Braak, C.G.H. Diks, B.A. Robinson, J.M. Hyman and D. Higdon. 2009. Accelerating Markov chain Monte Carlo simulation by Differential Evolution with Self-Adaptive Randomized Subspace Sampling. International Journal of Nonlinear Science \& Numerical Simulation, 10 (3), 271-288.

Vrugt , J.A. 2016., Markov chain Monte Carlo simulation using the DREAM software package: Theory, concepts, and Matlab implementation. Environmental Modelling \& Software, 75, 273-316

WAMDI group, 1988: The WAM model - a third generation ocean wave prediction model, J. Phys. Oceanogr., 18, 1775-1810 